\title{
Phenomenology of unpolarized TMDs from Semi-Inclusive DIS data
}

\author{
Andrea Signori ${ }^{* a, b}$, Alessandro Bacchetta, ${ }^{c, d}$ and Marco Radici ${ }^{d}$ \\ ${ }^{a}$ Department of Physics and Astronomy, VU University Amsterdam \\ De Boelelaan 1081, NL-1081 HV Amsterdam, the Netherlands \\ ${ }^{b}$ Nikhef \\ Science Park 105, NL-1098 XG Amsterdam, the Netherlands \\ c INFN Sezione di Pavia \\ via Bassi 6, I-27100 Pavia, Italy \\ ${ }^{d}$ Dipartimento di Fisica, Università di Pavia \\ via Bassi 6, I-27100 Pavia, Italy \\ E-mail: asignori@nikhef.nl, alessandro.bacchetta@unipv.it, \\ marco.radici@pv.infn.it
}

We discuss the dependence on the flavor of the intrinsic transverse momentum in unpolarized transverse-momentum-dependent distribution functions (TMD PDFs) and fragmentation functions (TMD FFs) analyzing data of semi-inclusive deep-inelastic scattering (SIDIS) released by the HERMES collaboration. Adopting a flavor-dependent Gaussian model in the transverse momentum and neglecting QCD evolution, we find interesting evidences concerning the flavor dependence in TMD FFs, whereas the indications are weaker in TMD PDFs and deserve further investigations. Inclusions of new data sets of SIDIS, $\mathrm{e}^{+} \mathrm{e}^{-}$annihilations and and Drell-Yan (DY) events require a proper treatment of QCD evolution. We try to get constraints on the nonperturbative Sudakov factor from $\mathrm{e}^{+} \mathrm{e}^{-}$annihilations into hadrons.

$$
\text { PACS: 13.60.-r, 13.87.Fh, 14.20.Dh, 14.65.Bt }
$$

XXII. International Workshop on Deep-Inelastic Scattering and Related Subjects 28 April - 2 May 2014

Warsaw, Poland

\footnotetext{
* Speaker.
} 


\section{Introduction and motivation}

TMDs are not phenomenologically well known. For example, even in the simple unpolarized distribution $f_{1}\left(x, k_{\perp}^{2}\right)$ (i.e., the distribution of partons with flavor $a$ summed over their polarization and averaged over the polarization of the parent hadron) the transverse momentum dependence is poorly known. This work refers to one of the first phenomenological investigations (see Refs. [1], [2]) of the unpolarized TMDs, addressing their flavor structure in the transverse momentum. For a list of physical motivations underlying this study see Ref. [1]. Above all, it is important to notice that the SIDIS data sets for different combinations of target and detected hadrons are different. Introducing the flavor dependence in the TMD part of distribution and fragmentation functions allows us to theoretically discriminate the available configurations. This is not possible in the simple flavor-independent Gaussian ansatz.

\section{Unpolarized SIDIS and flavor-dependent Gaussian TMDs}

In one-hadron inclusive DIS a lepton scatters off a nucleon $N$ and one hadron $h$ is identified in the final state:

$$
\ell+N \rightarrow \ell^{\prime}+h+X
$$

where $\ell, \ell^{\prime}$ denote the incoming and outgoing lepton respectively. The light-cone transverse momenta involved in the process are:

\begin{tabular}{ll} 
Momentum & Physical meaning \\
\hline $\boldsymbol{k}_{\perp}$ & intrinsic partonic transverse momentum \\
$\boldsymbol{P}_{\perp}$ & transverse momentum of final hadron w.r.t. fragmenting parton \\
$\boldsymbol{P}_{h T}$ & transverse momentum of final hadron w.r.t. virtual photon
\end{tabular}

We consider unpolarized scattering integrated over the azimuthal angle $\phi_{h}$ of the detected hadron in the limit of small transverse momenta and at leading twist (respectively, $\boldsymbol{P}_{h T}^{2} \ll Q^{2}$ and $M^{2} \ll Q^{2}$, being $Q^{2}$ the hard scale of the process and $M$ the mass of the target hadron). As a first investigation, we adopt the parton model picture performing a leading order QED analysis (one-photon exchange approximation) neglecting any modification that can be induced by QCD evolution. This is possible because of the limited range in $Q^{2}$ (see Refs. [3], [1]).

The HERMES collaboration released SIDIS multiplicities (see Ref. [3]), the differential number of hadrons produced per DIS event. Multiplicity is defined as the ratio of the SIDIS and the DIS cross sections and, at leading twist, is proportional to the unpolarized transverse structure function $F_{U U, T}$ (see Ref. [4]):

$$
m_{N}^{h}\left(x, z, Q^{2}, \boldsymbol{P}_{h T}^{2}\right)=\frac{d^{(4)} \sigma_{N}^{h} / d x d Q^{2} d z d \boldsymbol{P}_{h T}^{2}}{d^{(2)} \sigma_{\mathrm{DIS}} / d x d Q^{2}} \sim F_{U U, T}\left(x, z, Q^{2}, \boldsymbol{P}_{h T}^{2}\right)
$$

$F_{U U, T}$ is expressed in terms of a convolution of a TMD PDF and a TMD FF relying on the factorized formula for low transverse momentum SIDIS:

$$
F_{U U, T}\left(x, z, Q^{2}, \boldsymbol{P}_{h T}^{2}\right)=\sum_{a} e_{a}^{2}\left[f_{1}^{a, N}\left(x, \boldsymbol{k}_{\perp}^{2}, Q^{2}\right) \otimes D_{1}^{a \rightarrow h}\left(z, \boldsymbol{P}_{\perp}^{2}, Q^{2}\right)\right],
$$


where $a$ is the flavor index ${ }^{1}$ and the convolution is defined in Ref. [4]. A more extensive description of the assumptions, the notation, and the general theoretical framework is available in Ref. [1].

The flavor-dependent Gaussian hypothesis consists in assuming flavor-dependent Gaussian behavior in the transverse momentum in the unpolarized distribution function $f_{1}^{a, N}$ and fragmentation function $D_{1}^{a \rightarrow h}$ :

$$
f_{1}^{a, N}\left(x, Q^{2}, \boldsymbol{k}_{\perp}^{2}\right)=\frac{f_{1}^{a, N}\left(x, Q^{2}\right)}{\pi\left\langle\boldsymbol{k}_{\perp, a}^{2}\right\rangle} e^{-\boldsymbol{k}_{\perp}^{2} /\left\langle\boldsymbol{k}_{\perp, a}^{2}\right\rangle}, \quad D_{1}^{a \rightarrow h}\left(z, Q^{2}, \boldsymbol{P}_{\perp}^{2}\right)=\frac{D_{1}^{a \rightarrow h}\left(z, Q^{2}\right)}{\pi\left\langle\boldsymbol{P}_{\perp, a \rightarrow h}^{2}\right\rangle} e^{-\boldsymbol{P}_{\perp}^{2} /\left\langle\boldsymbol{P}_{\perp, a \rightarrow h}^{2}\right\rangle} .
$$

Introducing this assumption the multiplicity reads:

$$
\begin{aligned}
m_{N}^{h}\left(x, z, Q^{2}, \boldsymbol{P}_{h T}^{2}\right) & =\frac{\pi}{\sum_{a} e_{a}^{2} f_{1}^{a, N}\left(x, Q^{2}\right)} \\
& \times \sum_{a}\left[e_{a}^{2} f_{1}^{a, N}\left(x, Q^{2}\right) D_{1}^{a \rightarrow h}\left(z, Q^{2}\right) \frac{e^{-\frac{\boldsymbol{P}_{h T}^{2}}{z^{2}\left\langle\boldsymbol{k}_{\perp, a}^{2}\right\rangle+\left\langle\boldsymbol{P}_{\perp, a \rightarrow h}^{2}\right\rangle}}}{\pi\left(z^{2}\left\langle\boldsymbol{k}_{\perp, a}^{2}\right\rangle+\left\langle\boldsymbol{P}_{\perp, a \rightarrow h}^{2}\right\rangle\right)}\right],
\end{aligned}
$$

where the Gaussian functions in $\boldsymbol{P}_{h T}$ result from the convolution of $f_{1}^{a, N}$ and $D_{1}^{a \rightarrow h}$. The multiplicity is not a Gaussian in $\boldsymbol{P}_{h T}$, because it is a summation of Gaussians functions. This is important, since it experimentally deviates from the Gaussian functional form.

\section{Assumptions concerning average transverse momenta}

We adopt a simple framework for flavor dependence, introducing different widths for upvalence, down-valence and sea quarks, with a dependence on the longitudinal momentum fraction $x$. The latter is driven by data analysis, but above all it is suggested by theoretical considerations and model calculations (see Ref. [1]):

$$
\left\langle\boldsymbol{k}_{\perp, a}^{2}\right\rangle(x)=\left\langle\hat{\boldsymbol{k}}_{\perp, a}^{2}\right\rangle \frac{(1-x)^{\alpha} x^{\sigma}}{(1-\hat{x})^{\alpha} \hat{x}^{\sigma}}, \quad \text { where }\left\langle\hat{\boldsymbol{k}}_{\perp, a}^{2}\right\rangle \equiv\left\langle\boldsymbol{k}_{\perp, a}^{2}\right\rangle(\hat{x}), \text { and } \hat{x}=0.1 .
$$

$\left\langle\hat{\boldsymbol{k}}_{\perp, a}^{2}\right\rangle, \alpha, \sigma$ are free parameters: $\alpha$ and $\sigma$ are flavor-independent, whereas we have three flavordependent normalizations, $\left\langle\hat{\boldsymbol{k}}_{\perp, a}^{2}\right\rangle$ for $a=u_{v}, d_{v}$, sea. Concerning TMD FFs, we distinguish three favored process and one class of unfavored processes, assuming charge conjugation and isospin symmetry:

$$
\begin{aligned}
&\left\langle\boldsymbol{P}_{\perp, u \vdash \pi^{+}}^{2}\right\rangle=\left\langle\boldsymbol{P}_{\perp, \bar{d} \bullet \pi^{+}}^{2}\right\rangle=\left\langle\boldsymbol{P}_{\perp, \bar{l} \bullet \pi^{-}}^{2}\right\rangle=\left\langle\boldsymbol{P}_{\perp, d \bullet \pi^{-}}^{2}\right\rangle \equiv\left\langle\boldsymbol{P}_{\perp, \text { fav }}^{2}\right\rangle, \\
&\left\langle\boldsymbol{P}_{\perp, u \mapsto K^{+}}^{2}\right\rangle=\left\langle\boldsymbol{P}_{\perp, \bar{l} \bullet K^{-}}^{2}\right\rangle \equiv\left\langle\boldsymbol{P}_{\perp, u K}^{2}\right\rangle, \\
&\left\langle\boldsymbol{P}_{\perp, \bar{s} K^{+}}^{2}\right\rangle=\left\langle\boldsymbol{P}_{\perp, s \vdash K^{-}}^{2}\right\rangle \equiv\left\langle\boldsymbol{P}_{\perp, s K}^{2}\right\rangle, \\
&\left\langle\boldsymbol{P}_{\perp, \text { all others }}^{2}\right\rangle \equiv\left\langle\boldsymbol{P}_{\perp, \text { unf }}^{2}\right\rangle .
\end{aligned}
$$

\footnotetext{
${ }^{1}$ In summing over the flavor index up and down contributions will consist of two parts: one for valence quarks and one for sea quarks.
} 
As for the previous case, we introduce a dependence on the longitudinal momentum fraction $z$ in the variance of the Gaussian TMD FFs:

$$
\left\langle\boldsymbol{P}_{\perp, a \rightarrow h}^{2}\right\rangle(z)=\left\langle\hat{\boldsymbol{P}}_{\perp, a \rightarrow h}^{2}\right\rangle \frac{\left(z^{\beta}+\boldsymbol{\delta}\right)(1-z)^{\gamma}}{\left(\hat{z}^{\beta}+\boldsymbol{\delta}\right)(1-\hat{z})^{\gamma}} \quad \text { where }\left\langle\hat{\boldsymbol{P}}_{\perp, a \rightarrow h}^{2}\right\rangle \equiv\left\langle\boldsymbol{P}_{\perp, a \rightarrow h}^{2}\right\rangle(\hat{z}), \text { and } \hat{z}=0.5 .
$$

The kineamtic parameters $\beta, \gamma$ and $\delta$ are flavor-independent, whereas we have one normalization for each class of fragmentation processes.

\section{Phenomenology}

We fit the HERMES data sets related to proton and deuteron targets and pions and kaons in the final state, with multidimensional binning in $x, z$ and $Q^{2}$. COMPASS data will be considered in the future. Since the $Q^{2}$ range is narrow, we can carry out the analysis at a fixed $Q^{2}=2.4 \mathrm{GeV}^{2}$ in Eq. (2.5), neglecting any effect from QCD evolution. We fit $\mathscr{M}=200$ Monte Carlo replicas of the original data set, in order to get $\mathscr{M}$ best values for each parameter. A detailed description of the kinematic cuts on the data and the Monte Carlo fit procedure is given in Ref. [1].

We perform four fits with different assumptions. The first one, conventionally named default fit, is based on the less restrictive assumption for kinematic cuts. In the second one, we further exclude data with $Q^{2}<1.6 \mathrm{GeV}^{2}$. The third scenario corresponds to neglecting kaons in the final state. The last scenario is a fit of the default selection using a flavor-independent Gaussian ansatz. Here we will shortly present results related to the first and the fourth fits, the others being available in Ref. [1].

In the default fit the average global $\chi^{2} /$ d.o.f. is $1.63 \pm 0.13$. The quality of the fit is not excellent, but we need to take into account that the description of the multiplicities is difficult already in the collinear case (see Ref. [1]). In the flavor-independent case the agreement is slightly worse, with an average global $\chi^{2} /$ d.o.f. of $1.72 \pm 0.11$. This is because with the flavor-independent model we fit different data sets with the same model function.

In Tab. 1 and Tab. 2 we list the average best values for some ${ }^{2}$ of the fit parameters related to TMD PDFs and TMD FFs respectively. In the left panel of Fig. 1, we compare the ratio $\left\langle\boldsymbol{k}_{\perp, d_{v}}^{2}\right\rangle /\left\langle\boldsymbol{k}_{\perp, u_{v}}^{2}\right\rangle$ vs. $\left\langle\boldsymbol{k}_{\perp, \text { sea }}^{2}\right\rangle /\left\langle\boldsymbol{k}_{\perp, u_{v}}^{2}\right\rangle$ calculated within the 200 replicas. The dashed lines correspond to unitary ratios and divide the plane into four sectors. For many replicas, the values lie in the upper left quadrant, meaning that $\left\langle\boldsymbol{k}_{\perp, d_{v}}^{2}\right\rangle\left\langle\left\langle\boldsymbol{k}_{\perp, u_{v}}^{2}\right\rangle<\left\langle\boldsymbol{k}_{\perp, \text { sea }}^{2}\right\rangle\right.$. On average, $d_{v}$ is about $20 \%$ narrower than $u_{v}$, which is about $10 \%$ narrower than the sea. The crossing of the dashed lines corresponds to the flavor-independent configuration, in which all transverse momenta are equal. This point lies at the limit of the $68 \%$ confidence region, meaning that the flavor-independent configuration is not statistically ruled out by the Monte Carlo fit. In the right panel of Fig. 1, we compare the ratio $\left\langle\boldsymbol{P}_{\perp, \text { unf }}^{2}\right\rangle /\left\langle\boldsymbol{P}_{\perp, \text { fav }}^{2}\right\rangle$ vs. $\left\langle\boldsymbol{P}_{\perp, u K}^{2}\right\rangle /\left\langle\boldsymbol{P}_{\perp, \text { fav }}^{2}\right\rangle$. On average, the width of unfavored and $u \rightarrow K$ fragmentations are about $20 \%$ larger than the widht of favored ones. All points are concentrated in the upper right quadrant: we have the clear outcome that $\left\langle\boldsymbol{P}_{\perp, \text { fav }}^{2}\right\rangle\left\langle\left\langle\boldsymbol{P}_{\perp, \text { unf }}^{2}\right\rangle \sim\left\langle\boldsymbol{P}_{\perp, u K}^{2}\right\rangle\right.$ and that the flavor-independent configuration is well outside the $68 \%$ confidence region. Results from a flavor-independent Gaussian fit of HERMES and COMPASs data are also available in Ref. [5].

\footnotetext{
${ }^{2}$ for a complete list please see Ref. [1].
} 


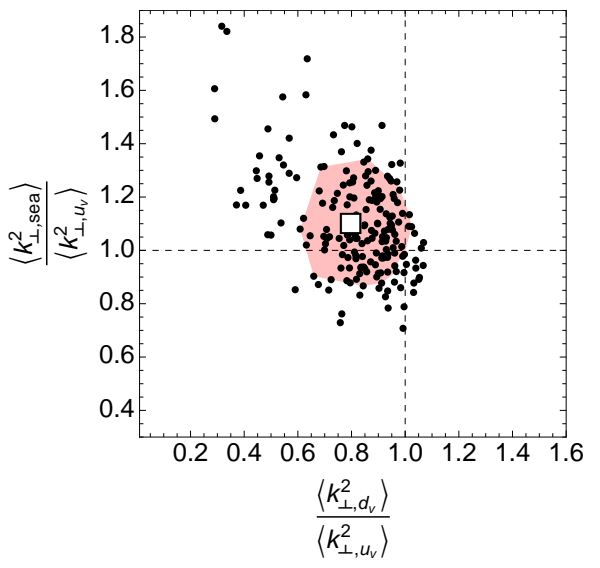

(a)

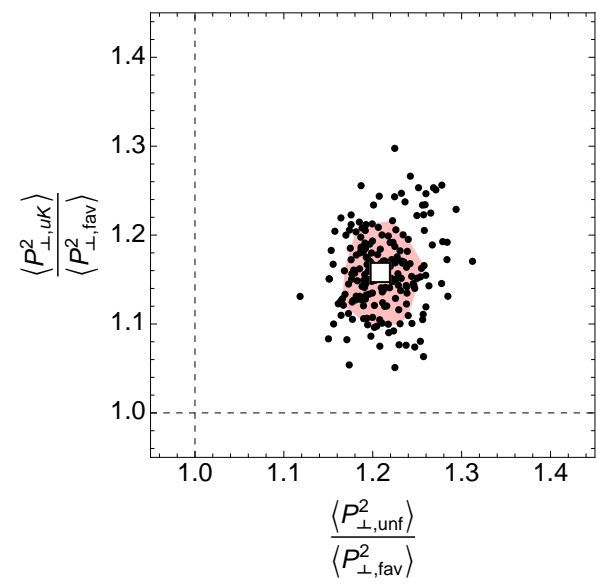

(b)

Figure 1: (a) Distribution of the values of the ratios $\left\langle\boldsymbol{k}_{\perp, d_{v}}^{2}\right\rangle /\left\langle\boldsymbol{k}_{\perp, u_{v}}^{2}\right\rangle$ vs. $\left\langle\boldsymbol{k}_{\perp, \text { sea }}^{2}\right\rangle /\left\langle\boldsymbol{k}_{\perp, u_{v}}^{2}\right\rangle$ obtained from fitting 200 replicas of the original data points. The white squared box indicates the center of the $68 \%$ confidence interval for each ratio. The shaded area represents the two-dimensional $68 \%$ confidence region around the white box. The dashed lines correspond to the ratios being unity; their crossing point corresponds to the result with no flavor dependence. For most of the points, $\left\langle\boldsymbol{k}_{\perp, d_{v}}^{2}\right\rangle\left\langle\left\langle\boldsymbol{k}_{\perp, u_{v}}^{2}\right\rangle\left\langle\left\langle\boldsymbol{k}_{\perp \text {,sea }}^{2}\right\rangle\right.\right.$. (b) Same as previous panel, but for the distribution of the values of the ratios $\left\langle\boldsymbol{P}_{\perp, \text { unf }}^{2}\right\rangle /\left\langle\boldsymbol{P}_{\perp, \text { fav }}^{2}\right\rangle$ vs. $\left\langle\boldsymbol{P}_{\perp, u K}^{2}\right\rangle /\left\langle\boldsymbol{P}_{\perp, \text { fav }}^{2}\right\rangle$. For all points, $\left\langle\boldsymbol{P}_{\perp, \text { fav }}^{2}\right\rangle\left\langle\left\langle\boldsymbol{P}_{\perp, \text { unf }}^{2}\right\rangle \sim\left\langle\boldsymbol{P}_{\perp, u K}^{2}\right\rangle\right.$.

\begin{tabular}{|c|c|c|c|}
\hline \multicolumn{4}{|c|}{ Parameters for TMD PDFs } \\
\hline \hline & $\left\langle\hat{\boldsymbol{k}}_{\perp, d_{v}}^{2}\right\rangle\left[\mathrm{GeV}^{2}\right]$ & $\left\langle\hat{\boldsymbol{k}}_{\perp, u_{v}}^{2}\right\rangle\left[\mathrm{GeV}^{2}\right]$ & $\left\langle\hat{\boldsymbol{k}}_{\perp, \text { sea }}^{2}\right\rangle\left[\mathrm{GeV}^{2}\right]$ \\
\hline \hline Default & $0.30 \pm 0.17$ & $0.36 \pm 0.14$ & $0.41 \pm 0.16$ \\
\hline Flavor-indep. & $0.30 \pm 0.10$ & $0.30 \pm 0.10$ & $0.30 \pm 0.10$ \\
\hline
\end{tabular}

Table 1: $68 \%$ confidence intervals of average mean square transverse momenta for TMD PDFs in the different scenarios.

\begin{tabular}{|c|c|c|c|c|}
\hline \multicolumn{5}{|c|}{ Parameters for TMD FFs } \\
\hline \hline & $\left\langle\hat{\boldsymbol{P}}_{\perp, \text { fav }}^{2}\right\rangle\left[\mathrm{GeV}^{2}\right]$ & $\left\langle\hat{\boldsymbol{P}}_{\perp, \text { unf }}^{2}\right\rangle\left[\mathrm{GeV}^{2}\right]$ & $\left\langle\hat{\boldsymbol{P}}_{\perp, s K}^{2}\right\rangle\left[\mathrm{GeV}^{2}\right]$ & $\left\langle\hat{\boldsymbol{P}}_{\perp, u K}^{2}\right\rangle\left[\mathrm{GeV}^{2}\right]$ \\
\hline \hline Default & $0.15 \pm 0.04$ & $0.19 \pm 0.04$ & $0.19 \pm 0.04$ & $0.18 \pm 0.05$ \\
\hline Flavor-indep. & $0.18 \pm 0.03$ & $0.18 \pm 0.03$ & $0.18 \pm 0.03$ & $0.18 \pm 0.03$ \\
\hline
\end{tabular}

Table 2: $68 \%$ confidence intervals of average mean square transverse momenta for TMD FFs in the different scenarios.

\section{Extensions}

In all the configurations we observe a strong anticorrelation between transverse momenta in distribution and fragmentation functions. This is not surprising, since the width of the observed $\boldsymbol{P}_{h T}$ distribution is given in Eq. (2.5) as a summation of partonic transverse momenta. To better constrain the transverse momenta in TMD PDFs and FFs it will be essential to include also data from electron-positron annihilations and Drell-Yan processes. In facing this task it is fundamental to introduce QCD evolution, to distinguish perturbative from non-perturbative components in 
partonic transverse momenta (see, e.g., Ref. [6] and [7]). Assuming to describe the soft initialscale transverse momenta with flavor-dependent Gaussian distributions, we still do not know the compatible values for the parameters modelling the soft contributions to the evolution. For this reason, we calculate the transverse-momentum-dependent cross section for $\mathrm{e}^{+} \mathrm{e}^{-}$annihilations into one and two hadrons, aiming to constrain the most physical configurations in the evolution from the comparison with forthcoming data (to be released by the BELLE and BABAR collaborations). At the same time, we want to pin down the most physical flavor-dependent replicas of Gaussian TMD FFs out of the 200 equivalent ones fitted on the HERMES data. Once we fix the parameters for the evolution compatible with our extracted TMD FFs, we will have a consistent framework to perform a global fit of different data sets.

\section{Conclusions}

We moved the first steps in the process of investigating the flavor dependence of partonic transverse momentum through unpolarized TMDs. The impact of this study, however, will go beyond unpolarized distributions: it will also affect the extractions of the polarized ones, because the unpolarized TMDs enter the denominator of spin asymmetries. Moreover, it will be interesting to test its effect on Monte Carlo generators sensitive to the intrinsic transverse momentum of partons (like GMCTRANS , Herwig, Pythia, ResBos, Cascade). To get a better phenomenological perspective we need to include more data in a framework which properly takes into account QCD evolution. Further papers will describe the achievements made along this way. This work is part of the program of the Stichting voor Fundamenteel Onderzoek der Materie (FOM), supported by the Nederlandse Organisatie voor Wetenschappelijk Onderzoek (NWO).

\section{References}

[1] A. Signori, A. Bacchetta, M. Radici and G. Schnell, Investigations into the flavor dependence of partonic transverse momentum, JHEP 1311, 194 (2013) [arXiv:1309.3507 [hep-ph], arXiv:1309.3507].

[2] A. Signori, A. Bacchetta and M. Radici, Flavor dependence of unpolarized TMDs from semi-inclusive pion production, Int. J. Mod. Phys. Conf. Ser. 25, 1460020 (2014) [arXiv:1309.5929 [hep-ph]].

[3] A. Airapetian et al. [HERMES Collaboration], Multiplicities of charged pions and kaons from semi-inclusive deep-inelastic scattering by the proton and the deuteron, Phys. Rev. D 87, 074029 (2013) [arXiv:1212.5407 [hep-ex]].

[4] A. Bacchetta, M. Diehl, K. Goeke, A. Metz, P. J. Mulders and M. Schlegel, Semi-inclusive deep inelastic scattering at small transverse momentum, JHEP 0702, 093 (2007) [hep-ph/0611265].

[5] M. Anselmino, M. Boglione, J. O. Gonzalez H., S. Melis and A. Prokudin, Unpolarised Transverse Momentum Dependent Distribution and Fragmentation Functions from SIDIS Multiplicities, JHEP 1404, 005 (2014) [arXiv:1312.6261 [hep-ph]].

[6] J. Collins, Foundations of perturbative QCD, (Cambridge monographs on particle physics, nuclear physics and cosmology. 32)

[7] M. G. Echevarriá, A. Idilbi, A. Schäfer and I. Scimemi, Model-Independent Evolution of Transverse Momentum Dependent Distribution Functions (TMDs) at NNLL, Eur. Phys. J. C 73, 2636 (2013) [arXiv:1208.1281 [hep-ph]]. 